\title{
Plasmonic Properties of Silver Amalgam Nanoparticles Studied by Analytical Transmission Electron Microscopy
}

Michal Horák ${ }^{1}$, Filip Ligmajer ${ }^{2}$, Tomáš Šikola ${ }^{2}$, Miroslav Fojta ${ }^{3}$ and Aleš Daňhel ${ }^{4}$

${ }^{1}$ CEITEC Brno University of Technology, Brno, Jihomoravsky kraj, Czech Republic, ${ }^{2}$ CEITEC Brno University of Technology and Institute of Physical Engineering, Brno University of Technology, Brno, Jihomoravsky kraj, Czech Republic, ${ }^{3}$ Institute of Biophysics, Czech Academy of Sciences and CEITEC Masaryk University, Brno, Jihomoravsky kraj, Czech Republic, ${ }^{4}$ Institute of Biophysics, Czech Academy of Sciences, Brno, Jihomoravsky kraj, Czech Republic

Silver amalgam is one of the most suitable solid electrode materials in electroanalysis of various reducible organic and inorganic compounds including heavy metals, agrochemicals, colorants, drugs, environmental pollutants, and biologically important compounds [1]. The main advantage of silver amalgam within this context is its wide cathodic potential window, high mechanical stability, adequate sensitivity, and advantageous strong interaction with biopolymers (e.g. DNA and proteins) [2]. When such material is applied to the conventional electrodes, it helps to increase its active surface area, charge transfer efficiency and overall sensitivity, though it also introduces additional complexity to the system. Nanostructuring the silver amalgam promises improved electrochemical performance and brings along the prospect of plasmonic activity. However, there has been a lack of knowledge about the optical properties of nanostructured or even bulk silver amalgam which prevents its using in spectroelectrochemical and photochemical studies. We present a study of optical properties of silver amalgam and demonstrate the plasmonic nature of its nanoparticles [3] as they support localized surface plasmon resonances (LSPR), which are self-sustained collective oscillations of free electrons in metal nano- and microstructures.

Prior the single-particle level investigation by analytical (scanning) transmission electron microscopy (S/TEM), we have studied their optical response using Fourier-transform infrared spectrometry and spectroscopic ellipsometry. Optical measurements have demonstrated the plasmon resonance of sufficient quality for spectroelectrochemistry ranging from ultraviolet to mid-infrared region and they gave us the dielectric function of a thick silver amalgam layer. To understand the nature of plasmonic modes in silver amalgam particles, we have focused on studying single nanoparticles by TEM including energy dispersive X-ray spectroscopy (EDS) for material analysis and electron energy loss spectroscopy (EELS) for measuring the surface plasmon resonances. Silver amalgam nanoparticles were prepared by controlled electrodeposition on a conductive indium tin oxide (ITO) support from a mixed solution of silver and mercury ions [4]. In order to prepare the samples for TEM, silver amalgam nanoparticles were washed off the ITO into demineralized water in an ultrasonic bath and the resulting solution was dropped and dried on a standard silicon nitride membrane for TEM.

First, we have studied the morphology, crystallography, and chemical composition of silver amalgam nanoparticles. Figure 1(a) shows the TEM micrograph of a typical silver amalgam rod-shaped nanoparticle. This nanoparticle is polycrystalline, which was confirmed by selected area diffraction pattern shown in Figure 1(b). Figure 1(c) shows the high-angle annular dark field micrograph and elemental maps showing the chemical composition of a silver amalgam nanoparticle on a silicon nitride membrane. It reads in weight percents $55 \%$ of $\mathrm{Ag}$ and $45 \%$ of $\mathrm{Hg}$ in the case of the particle, and $90 \%$ of $\mathrm{Ag}$ and $10 \%$ of $\mathrm{Hg}$ in the case of the small satellite, respectively.

Second, we have studied the optical properties of silver amalgam nanoparticles represented by LSPR. The rod-shaped silver amalgam nanoparticle presented in Figure 2 exhibits three different resonant modes 
which were also complemented by the numerical simulations using boundary element method (BEM): the longitudinal dipole at $1.77 \mathrm{eV}$, the transverse dipole mode at $2.8 \mathrm{eV}$, and the quadrupole mode at $3.2 \mathrm{eV}$. Moreover, we have proved that by changing the size of the nanoparticle the energy of dipole LSPR mode can be tuned from ultraviolet to mid-infrared region.

To conclude, our results show that silver amalgam, apart from its proven usefulness in electroanalytic chemistry, can be also regarded as a novel plasmonic material with promising optical properties. Silver amalgam nano- and micro-particles exhibit strong plasmon resonances in ultraviolet to mid-infrared regions depending on the particle size. These findings establish silver amalgam nanoparticles as promising candidates for applications within photochemistry and spectroelectrochemistry, where the synergy between their plasmonic and electrochemical qualities can be fully utilized [5].
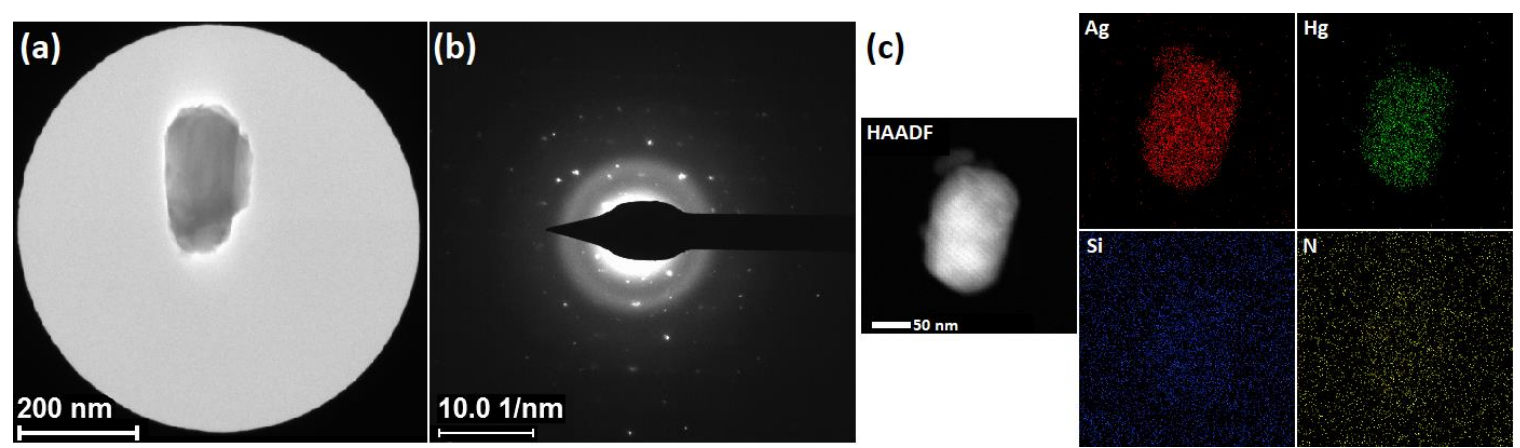

Figure 1. TEM micrograph (a) and selected area diffraction pattern (b) of a silver amalgam rod-shaped nanoparticle. STEM high-angle annular dark field (HAADF) micrograph and elemental maps showing the chemical composition of another rod-shaped silver amalgam nanoparticle on a silicon nitride membrane (c).

(a)
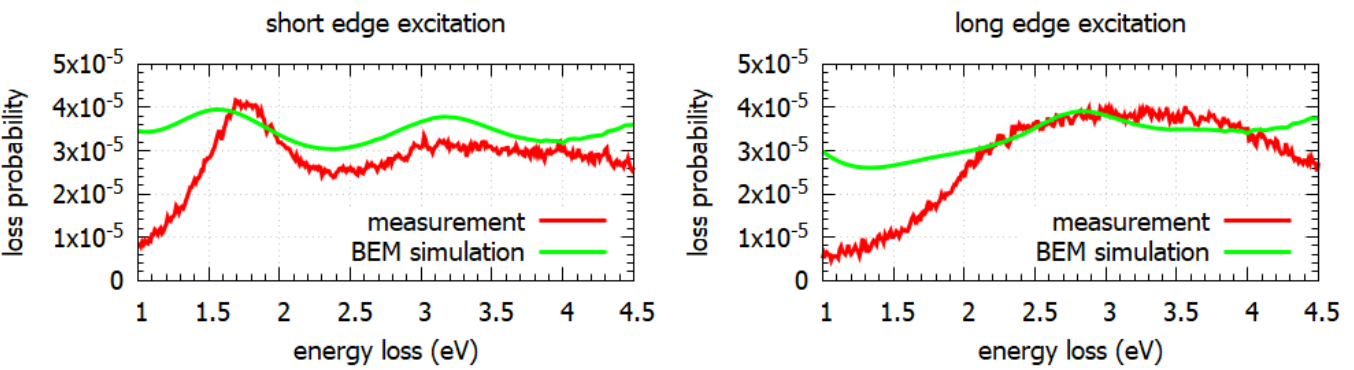

(b)

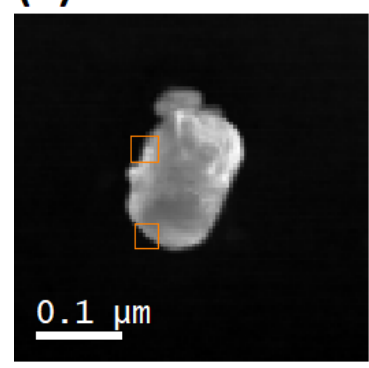

$(1.77 \pm 0.05) \mathrm{eV}$
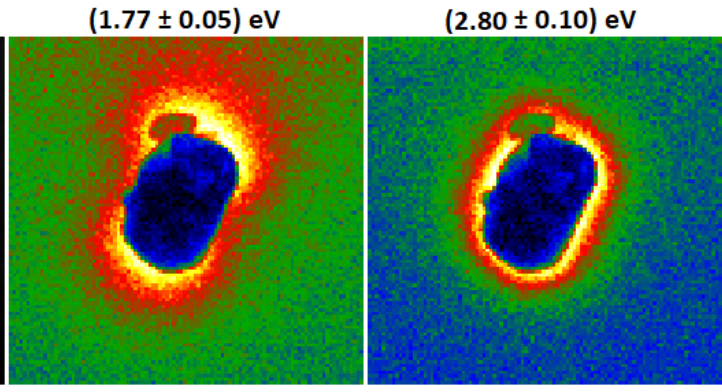

$(3.20 \pm 0.10) \mathrm{eV}$

Figure 2. Silver amalgam rod-shaped nanoparticle approximated by a rod with the length of $170 \mathrm{~nm}$, the width of $120 \mathrm{~nm}$, and the thickness of $80 \mathrm{~nm}$ : (a) background subtracted EEL spectra integrated over the orange squares in (b) complemented by numerical simulation corresponding to the excitation in the middle of the short edge and in the middle of the long edge of the particle; (b) STEM-HAADF micrograph and 
measured EEL maps at the energy of the peaks in (a) corresponding to the longitudinal dipole mode at $1.77 \mathrm{eV}$, the transverse dipole mode at $2.8 \mathrm{eV}$, and the quadrupole mode at $3.2 \mathrm{eV}$.

References

[1] A. Daňhel, J. Barek, Amalgam electrodes in organic electrochemistry, Curr. Org. Chem. 15 (2011) 2957-2969, doi: 10.2174/138527211798357218.

[2] P. Havranová, F. Ligmajer, A. Daňhel: Electrodeposition of silver amalgam on thin gold film electrodes for voltammetric detection of 4-nitrophenol and DNA labeled with osmium tetroxidebipyridine complex, Electroanalysis 31 (2019) 1952, doi: 10.1002/elan.201900306.

[3] F. Ligmajer, M. Horák, T. Šikola, M. Fojta, A. Daňhel, Silver amalgam nanoparticles: A novel plasmonic platform for spectroelectrochemistry, J. Phys. Chem. C 123 (2019) 16957-16964, doi: 10.1021/acs.jpcc.9b04124.

[4] A. Daňhel, F. Ligmajer, T. Šikola, A. Walcarius, M. Fojta, Electrodeposition of silver amalgam particles on ITO - towards novel electrode material, J. Electroanal. Chem. 821 (2018) 53-59, doi: 10.1016/j.jelechem.2017.12.008.

[5] Supported by Czech Science Foundation (17-23634Y), ERDF under the SYMBIT project (CZ.02.1.01/0.0/0.0/15_003/0000477), MEYS CR under the projects CzechNanoLab (LM2018110, 20202022) supporting the CEITEC Nano Research Infrastructure and CEITEC 2020 (LQ1601), and Brno University of Technology (CEITEC VUT-J-19-5945). M. H. acknowledges the support of Thermo Fisher Scientific and CSMS scholarship 2019. 